PRA-HEP/95-1

\title{
On the role of the quark mass thresholds in extrapolations of the running $\alpha_{s}$
}

\author{
Jiři Chýla \\ Institute of Physics, Academy of Sciences of the Czech Republic \\ Na Slovance 2, Prague 8, 18040 Czech Republic
}

\begin{abstract}
The accuracy of the conventional treatment of quark mass thresholds in the QCD running coupling constant $\alpha_{s}$, based on the step approximation to the $\beta$-function, is investigated. The errors of extrapolating $\alpha_{s}(\mu)$ from low energies to $\mu=M_{Z}$, implied by this approximation, are shown to be of the same magnitude as the typical next-to-next-to-leading order contributions to $\alpha_{s}$ and tend to increase the resulting $\alpha_{s}$. The importance of the proper choice of matching points is emphasized.
\end{abstract}

In this note I shall discuss the importance of the proper treatment of quark mass effects on the QCD running coupling constant $\alpha_{s}$. Although the quark mass effects are not large, steady improvement in the precision of experimental data, coming in particular from a new generation of experiments at CERN and Fermilab, combined with significant progress in higher order QCD calculations, have led to a renewed interest in quantitative aspects of these effects [1, 2]. There are essentially two reasons for it.

The first concerns the exploitation of the next-to-next-to-leading-order (NNLO) QCD calculation that have recently become available for a number of physical quantities [3, 4, 5, 6] and which exist basically for massless quarks only. The NNLO corrections are tiny effects and to include them makes sense only if they are large compared to errors resulting from the approximate treatment of the quark mass thresholds in massless QCD. To quantify the importance of the NNLO correction to the couplant $\alpha_{s}$, consider the difference between the values of $\alpha_{s}\left(\overline{\mathrm{MS}}, M_{Z}\right)$ in the NLO and NNLO approximations, taking, as an example, $\Lambda_{\frac{(5)}{\mathrm{MS}}}=0.2 \mathrm{GeV}$. At the $\mathrm{NLO}$ the couplant $a \equiv \alpha_{s} / \pi$ is given as a solution to the equation

$$
b \ln \frac{\mu}{\Lambda^{(5)}}=\frac{1}{a}+c \ln \frac{c a}{1+c a}
$$

where for $n_{f}$ massless quarks

$$
b=\frac{33-2 n_{f}}{6} ; \quad c=\frac{153-19 n_{f}}{33-4 n_{f}}
$$

are the first two, universal, coefficients of the QCD $\beta$-function appearing on the r.h.s. of the definition equation for the couplant $a(\mu)$

$$
\frac{\mathrm{d} a(\mu)}{\mathrm{d} \ln \mu} \equiv \beta(a)=-b a^{2}(\mu)\left(1+c a(\mu)+c_{2} a^{2}(\mu)+\cdots\right)
$$

For $\mu=M_{Z}, n_{f}=5$ and $\Lambda \frac{(5)}{\mathrm{MS}}=0.2 \mathrm{GeV}$ we get $a^{\mathrm{NLO}}\left(\overline{\mathrm{MS}}, M_{Z}\right)=0.03742$. At the NNLO and for $c_{2}>c^{2} / 4$ we have instead of (1)

$$
b \ln \frac{\mu}{\Lambda}=\frac{1}{a}+c \ln \frac{c a}{\sqrt{1+c a+c_{2} a^{2}}}+f\left(a, c_{2}\right)
$$




$$
f\left(a, c_{2}\right)=\frac{2 c_{2}-c^{2}}{d}\left(\arctan \frac{2 c_{2} a+c}{d}-\arctan \frac{c}{d}\right) ; \quad d \equiv \sqrt{4 c_{2}-c^{2}}
$$

where $c_{2}\left(\overline{\mathrm{MS}}, n_{f}=5\right)=1.475$. Combining (4) and (5) yields $a^{\mathrm{NNLO}}\left(\overline{\mathrm{MS}}, M_{Z}\right)=0.03665$. The relative difference between these two approximations

$$
\frac{a^{\mathrm{NLO}}-a^{\mathrm{NNLO}}}{a^{\mathrm{NLO}}} \doteq 0.02
$$

thus amounts to about $2 \%$. The NNLO correction should therefore be included only if the neglected effects can be expected to be smaller than this number. However, as we shall see, quark mass threshold effects can in some circumstances be just of this magnitude! In general the magnitude of higher order corrections to $\alpha_{s}$ depends on the renormalization scheme employed and so does also the estimate (6). However, if defined as the relative difference between the NLO and NNLO approximations this dependence is weak.

The second reason is related to the problem of comparing the values of $\alpha_{s}$, determined from different quantities characterized by vastly different momentum scales. As recently emphasized in an extensive review on $\alpha_{s}$ determinations [7], there is small, but nonnegligible discrepancy between the value of $\alpha_{s}\left(\overline{\mathrm{MS}}, M_{Z}\right)$ obtained by extrapolation from some of the low energy quantities and $\alpha_{s}\left(\overline{\mathrm{MS}}, M_{Z}\right)$ determined directly at the scale $M_{Z}$ at LEP, the latter giving the value higher by about $5-10 \%$. Simultaneously, it has been noted in [7] that there is an exception to this behaviour in the case of the ratio $R_{\tau}$ [B], which, when extrapolated from $m_{\tau}$ to $M_{Z}$, gives values of $\alpha_{s}$ close to that measured directly at LEP $(0.120 \pm 0.005$ [9]) as well. The physical relevance of the discrepancy between the low energy extrapolations and direct measurements of $\alpha_{s}$ at LEP has very recently been emphasized by Schifman in [10]. In particular, he argues that the extrapolations of $\alpha_{s}\left(m_{\tau}\right)$ to $\alpha_{s}\left(M_{Z}\right)$ are unreliable due to problems with the control of power corrections. As will be shown below, a part of this overestimate of the extrapolated value of $\alpha_{s}\left(M_{Z}\right)$ may in fact be simply due to the approximate treatment of the $c$ and $b$ quark thresholds.

In the rest of this note I shall analyze the quantitative consequences of the exact treatment of quarks mass thresholds at the LO and formulate the conventional matching procedure [11] for massless quarks in such a way that its results are so close to the exact ones that the available NNLO calculation can be consistently included. I shall describe in detail the approximation in which the "light" quarks $u, d$ and $s$ are considered massless, while the $c, b$ and $t$ quarks remain massive.

As complete multiloop calculations with massive quarks are very complicated and are available only at the leading order, all higher order phenomenological analyses use the calculations with a fixed effective number $n_{f}$ of massless quarks, depending on the characteristic scale of the quantity. In order to relate two regions of different effective numbers of massless quarks the approximate matching procedure developed in [11] is commonly used. It should be emphasized that this procedure concerns only those mass effects that can be absorbed in the renormalized couplant. At higher orders there are, however, mass effects that remain in the expansion coefficients even after the effects of heavy quarks have been absorbed in a suitably defined running couplant.

In QCD with (at least some) massive quarks the renormalization group equation for the couplant $a(\mu)$ looks formally as in massless QCD. The only, but important, difference concerns the two lowest order $\beta$-function coefficients, $b, b c$, which are no longer unique as in massless QCD, but may depend on the scale $\mu$. While in the class of $\overline{\mathrm{MS}}$-like renormalization conventions $b=11 / 2-n_{f} / 3$ as in massless QCD, in MOM-like ones it becomes a nontrivial function of the scale $\mu$ [12]

$$
b\left(\mu / m_{i}\right)=\frac{11}{2}-\frac{1}{3} \sum_{i} h_{i}\left(x_{i}\right), \quad x_{i} \equiv \frac{\mu}{m_{i}}
$$

where the sum runs over all the quarks considered, $m_{i}$ are the corresponding renormalized quark 

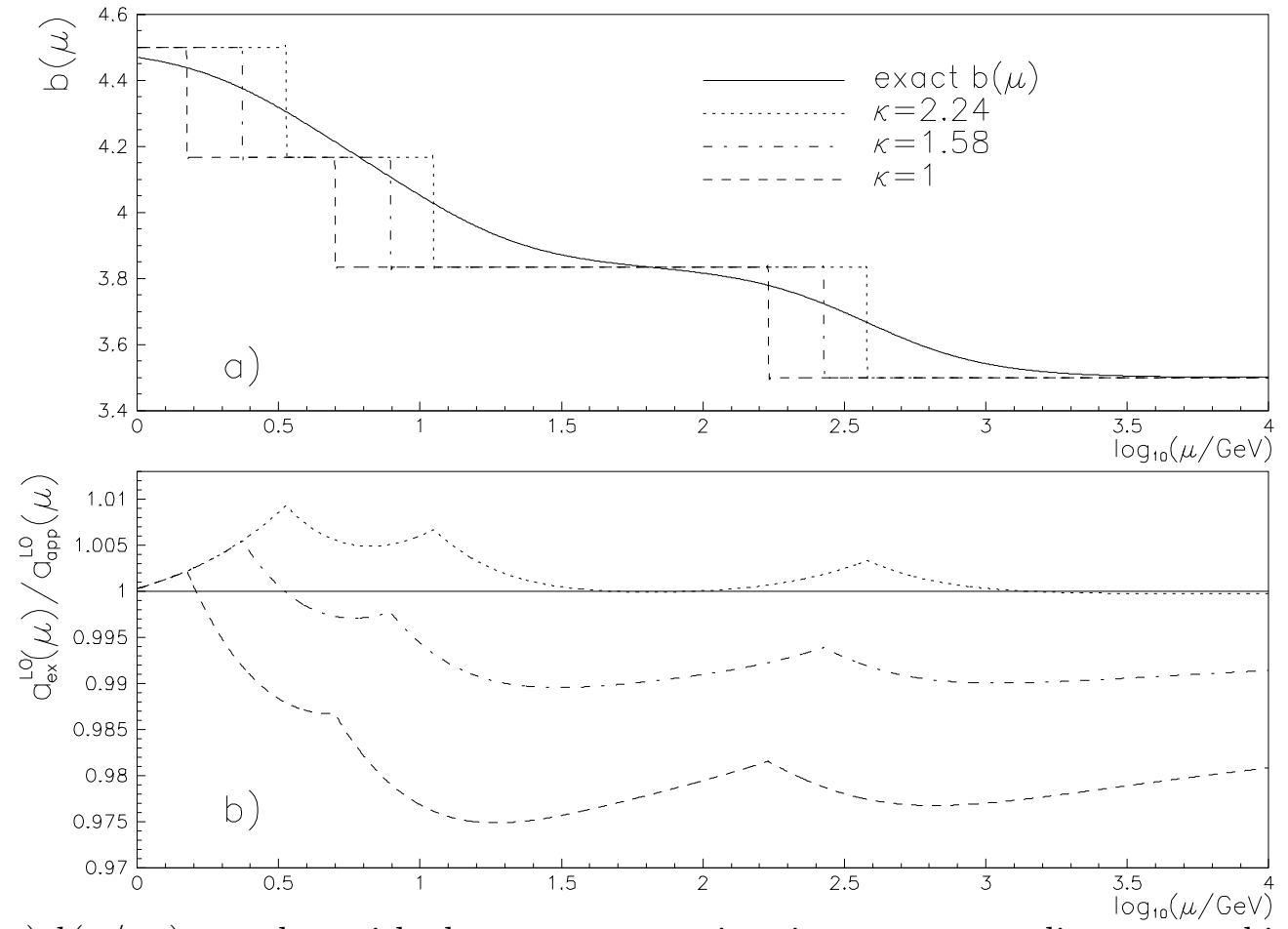

Figure 1: a) $b\left(\mu / m_{i}\right)$ together with three step approximations, corresponding to matching at the points $\mu_{i}=\kappa m_{i} ; i=c, b, t$ with $\left.\kappa=1,1.58,2.24 ; \mathrm{b}\right)$ the ratio $R_{a}$ for the same three approximations, made to coincide at $\mu_{0}=1 \mathrm{GeV}$.

masses ${ }^{1}$ and the threshold function $h(x)$ is given as [12]

$$
h(x) \equiv 6 x^{2} \int_{0}^{1} \mathrm{~d} z \frac{z^{2}(1-z)^{2}}{1+x^{2} z(1-z)}=1-\frac{6}{x^{2}}+\frac{12}{x^{3} \sqrt{4+x^{2}}} \ln \frac{\sqrt{4+x^{2}}+x}{\sqrt{4+x^{2}}-x} \doteq \frac{x^{2}}{5+x^{2}} .
$$

The last - approximate - equality is a very accurate approximation to the exact form of $h(x)$ in the whole range $x \in(0, \infty)$ [12]. This allows a simple treatment of the quark mass thresholds at the LO. There is no analogous calculation of the next $\beta$-function coefficient, $c$, for massive quarks and thus no possibility to repeat the present analysis at the NLO. This is one of the reasons why most of the phenomenological analyses use the so called "step" approximation in which at any value of $\mu$ one works with a finite effective number of massless quarks, which changes discontinuously at some matching points $\mu_{i}$. Consequently $b\left(n_{f}\right)$ becomes effectively a function of $\mu$, discontinuous at these matching points, as shown in the Fig.1a.

The matching points are assumed proportional to the masses of the corresponding quarks, $\mu_{i} \equiv \kappa m_{i}$. In principle different quark threshold could be associated with different $\kappa$, but for simplicity I take them equal. The free parameter $\kappa$, allowing for the variation of the proportionality factor, turns out to be quite important for the accuracy of the step approximation. At the LO the matching procedure then consists in the following relations at the matching points $\mu_{i}$ (the number in the superscript defines the corresponding effective number of massless quarks)

$$
\begin{aligned}
& a_{a p p}^{\mathrm{LO}, 3}\left(\kappa m_{c} / \Lambda^{(3)}\right)=a_{a p p}^{\mathrm{LO}, 4}\left(\kappa m_{c} / \Lambda^{(4)}\right) \Rightarrow \Lambda^{(4)}=\Lambda^{(3)}\left(\frac{\Lambda^{(3)}}{\kappa m_{c}}\right)^{1 / 3 b(4)} \\
& a_{a p p}^{\mathrm{LO}, 4}\left(\kappa m_{b} / \Lambda^{(4)}\right)=a_{a p p}^{\mathrm{LO}, 5}\left(\kappa m_{b} / \Lambda^{(5)}\right) \Rightarrow \Lambda^{(5)}=\Lambda^{(4)}\left(\frac{\Lambda^{(4)}}{\kappa m_{b}}\right)^{1 / 3 b(5)}
\end{aligned}
$$

\footnotetext{
${ }^{1}$ For the purpose of this discussion quark masses are regarded as constants, altough they too get renormalized and thus "run" as well.
} 


$$
a_{a p p}^{\mathrm{LO}, 5}\left(\kappa m_{t} / \Lambda^{(5)}\right)=a_{a p p}^{\mathrm{LO}, 6}\left(\kappa m_{t} / \Lambda^{(6)}\right) \Rightarrow \Lambda^{(6)}=\Lambda^{(5)}\left(\frac{\Lambda^{(5)}}{\kappa m_{t}}\right)^{1 / 3 b(6)}
$$

Note that each of the intervals of fixed $n_{f}$ is associated with a different value of the $\Lambda$-parameter, $\Lambda^{\left(n_{f}\right)}$. The resulting dependence $a\left(\mu / m_{i}\right)$ on $\mu$ is thus continuous at each of the matching points, but its derivatives at these points are discontinuous, reflecting the discontinuity of the step approximations to $b\left(\mu / m_{i}\right)$. This procedure can be easily extended to any finite order. Let me point out that the more sophisticated procedure for matching the couplants corresponding to different effective $n_{f}$ developed in [13] coincides to the LO with (9)-(11).

To estimate the errors in $\alpha_{s}$ resulting from the above defined approximate treatment of quark thresholds we merely need solve the LO equation with exact explicit mass dependence as given in (7)

$$
\frac{\mathrm{d} a(\mu)}{\mathrm{d} \ln \mu}=-a^{2}\left(\frac{11}{2}-\frac{1}{3} \sum_{i=1}^{6} h\left(x_{i}\right)\right) .
$$

For our purposes the approximation $h(x) \doteq x^{2} /\left(5+x^{2}\right)$ is entirely adequate and yields

$$
a(\mu)=\frac{1}{\left(\frac{11}{2}-\frac{3}{3}\right) \ln \frac{\mu}{\Lambda^{(3)}}-\frac{1}{3} \sum_{i=c, b, t} \ln \frac{\sqrt{\mu^{2}+5 m_{i}^{2}}}{\sqrt{\left(\Lambda^{(3)}\right)^{2}+5 m_{i}^{2}}}}
$$

where the fraction $\frac{3}{3}$ comes from the sum over the three massless quarks $u, d, s$ and $\Lambda^{(3)}$ is the corresponding $\Lambda$-parameter appropriate to 3 massless quarks. For the heavy quarks $c, b$ and $t \mathrm{I}$ take in the following $m_{c}=1.5 \mathrm{GeV}, m_{b}=5 \mathrm{GeV}, m_{t}=170 \mathrm{GeV}$. The distinction between the "light" and "heavy" quarks is given by the relative magnitude of $m_{i}$ and $\Lambda$, the latter being defined by the condition $5 m_{i}^{2} \gg \Lambda$. For the above values of $m_{c}, m_{b}, m_{t}$ this condition is very well satisfied. Consequently, for $\mu \ll m_{i}, i=c, b, t$ (13) approaches smoothly $a^{\mathrm{LO}}$ for $n_{f}=3$, while for $\mu \gg m_{i}$ and neglecting $\Lambda^{(3)}$ with respect to $5 m_{i}^{2}$, it goes to

$$
a(\mu)=\frac{1}{b(6) \ln \frac{\mu}{\Lambda^{(3)}}+\frac{1}{3} \ln \left(\frac{\sqrt{5} m_{c}}{\Lambda^{(3)}} \frac{\sqrt{5} m_{b}}{\Lambda^{(3)}} \frac{\sqrt{5} m_{t}}{\Lambda^{(3)}}\right)}=\frac{1}{b(6) \ln \frac{\mu}{\Lambda^{(6)}(\sqrt{5})}}
$$

where the parameter $\Lambda^{(6)}(\kappa)$ depends in general on $\kappa$ and

$$
\Lambda^{(6)}(\sqrt{5}) \equiv \Lambda^{(3)}\left(\frac{\Lambda^{(3)}}{\sqrt{5} m_{c}} \frac{\Lambda^{(3)}}{\sqrt{5} m_{b}} \frac{\Lambda^{(3)}}{\sqrt{5} m_{t}}\right)^{\frac{1}{3 b(6)}}=\left(\frac{1}{\sqrt{5}}\right)^{\frac{1}{b(6)}} \Lambda^{(6)}(1)
$$

coincides with $\Lambda^{(6)}$ defined via the subsequent application of the matching relations (99)-(11) for $\kappa=\sqrt{5} \doteq 2.24$. Although from the point of view of the matching procedure $\kappa$ is not exactly fixed, the value $\kappa=\sqrt{5}$ will be shown to be in some sense the best choice. The relation between $\Lambda^{(6)}$ and $\Lambda^{(3)}$ depends nontrivially on $\kappa$.

In Fig. 1a $b(\mu)$ is plotted as a function of $\mu$ for the above mentioned masses of $c, b$ and $t$ quarks, together with its step approximations corresponding to three different values of $\kappa=1, \sqrt{5}, \sqrt{5 / 2}$. There is hardly any sign of the steplike behaviour of the function $h(x)$ in the region of the $c$ and $b$ quark thresholds and only a very unpronounced indication of the plateau between the $b$ and $t$ quark thresholds. The steplike approximations are poor representations of the exact $h(x)$ primarily due to the rather slow approach of $h(x)$ to unity as $x \rightarrow \infty$. However, there is a marked difference between the three approximations. While the step approximation with the conventional choice 
$\kappa=1$ understimates the true $h(x)$ in the whole interval displayed and would do so even when some smoothing were applied, $\kappa=\sqrt{5}$ gives clearly much better approximation as the corresponding curve is intersected by the exact $h(x)$ at about the middle of each step.

In Fig. $1 \mathrm{~b}$ the $\mu$ dependence of the ratio

$$
R_{a} \equiv \frac{a_{e x}^{\mathrm{LO}}(\mu)}{a_{a p p}^{\mathrm{LO}}(\mu)}
$$

between the above exact solution (14) and the approximate expressions for the above mentioned values of $\kappa$ is plotted assuming $\Lambda^{(3)}=200 \mathrm{MeV}$. As we basicaly want to compare the results of different extrapolations starting from the same initial $\mu_{0}, \Lambda^{(3)}$ used in the approximate solutions was rescaled by the factor 1.004 with respect to $\Lambda^{(3)}$ in (13), thereby guaranteeing that all expressions coincide at $\mu_{0}=1 \mathrm{GeV}$. Any deviation from unity in Fig. $1 \mathrm{~b}$ is then entirely the effect of an approximate treatment of the heavy quark thresholds. The Fig.1 contains several simple messages.

The approximate solutions based on the matching procedure defined in (9)-(11) are in general much better immediately below the matching point than above it and is worst at about $5 m_{\text {match }}$. This reflects the fact that the function $h(x)$ vanishes fast (like $x^{2}$ ) at zero but approaches unity only very slowly. Moreover, in the $M_{Z}$ range the effect of the $c$ quark threshold is essentially the same as that of the $b$ quark and both are much more important than that of the top quark, although $M_{Z} / m_{c} \approx 60, M_{Z} / m_{b} \approx 18$, while $M_{Z} / m_{t} \approx 1 / 2$ !

The effect of varying $\kappa$ is quite important, in particular with respect to the $c$ and $b$ quark thresholds. In general $\kappa>1$ improves the approximation above the matching point, but worsens it immediately below it. The choice $\kappa=\sqrt{5}$, suggested by the asymptotic behaviour of (14) is clearly superior practically in the whole displayed interval $\mu \in\left(1,10^{4}\right) \mathrm{GeV}$ and leads to an excellent (on the level of $0.1 \%$ ) agreement with the exact solution in this interval. On the contrary the conventional choice $\kappa=1$ leads to much larger deviation from the exact result, which exceeds $2 \%$ in most of this region. This discrepancy is thus of the same magnitude as the effects of NNLO corrections to the couplant itself. It turns out that the effect of an exact treatment of the quark mass thresholds is as important as that of the NNLO correction to the $\beta$-function and must therefore be taken into account, whenever the latter is considered.

In Fig. 1 the exact as well as approximate expressions for the couplant are made to concide at $\mu_{0}=1 \mathrm{GeV}$, in order to stay below the $c$ quark threshold. In actual extrapolations one usually starts at somewhat higher scales. For the $\tau$-lepton decay rate as well as the GLS sume rule, the corresponding initial $\mu_{0}$ is about $2 \mathrm{GeV}$, for the various $\Upsilon$ characteristics $\mu_{0} \approx 10 \mathrm{GeV}$. In Fig. 2 the ratio (16) is therefore plotted for these two starting scales and the indicated values of $\kappa$. We see that

- For $\mu_{0}=2 \mathrm{GeV}$ the situation is essentially the same as for $\mu_{0}=1 \mathrm{GeV}$, i.e. extrapolated $\alpha_{s}^{\mathrm{LO}}\left(M_{Z}\right)$ is about $2 \%$ higher when the step approximation with $\kappa=1$ is used. The fact that conventional extrapolations of $\alpha_{s}\left(m_{\tau}\right)$ to $\alpha_{s}\left(M_{Z}\right)$ use this step approximation with $\kappa=1$ could thus cause part of the overestimate of $\alpha_{s}\left(M_{Z}\right)$ discussed in [10]. On the other hand the choice $\kappa=\sqrt{5}$ again leads to an excellent agreement with the exact extrapolation above 10 $\mathrm{GeV}$ and very satisfactory even down to $1 \mathrm{GeV}$.

- For $\mu_{0}=10 \mathrm{GeV}$ the choice $\kappa=\sqrt{5}$ is only marginally better that $\kappa=1$ when extrapolating to $\mu=M_{Z}$, but now both values yield $\alpha_{s}^{\mathrm{LO}}\left(M_{Z}\right)$ only about $0.5 \%$ from the exact LO result. This observation is consistent with the fact that $\alpha_{s}\left(M_{Z}\right)$ extrapolated from $\Upsilon$ characteristics is lower than that from $R_{\tau}$ and consistent with those based on deep inelastic scattering. For this choice of $\mu_{0}$ the superiority of the choice $\kappa=\sqrt{5}$ is obvious when extrapolating back, i.e. to lower scales $\mu$. Indeed, this is to be expected from Fig. 1b and 2a.

\footnotetext{
${ }^{2}$ The resulting ratio $R_{a}\left(M_{Z}\right)$ depends, however, only weekly on $\Lambda^{(3)}$ in the interval $(200,400) \mathrm{MeV}$.
} 


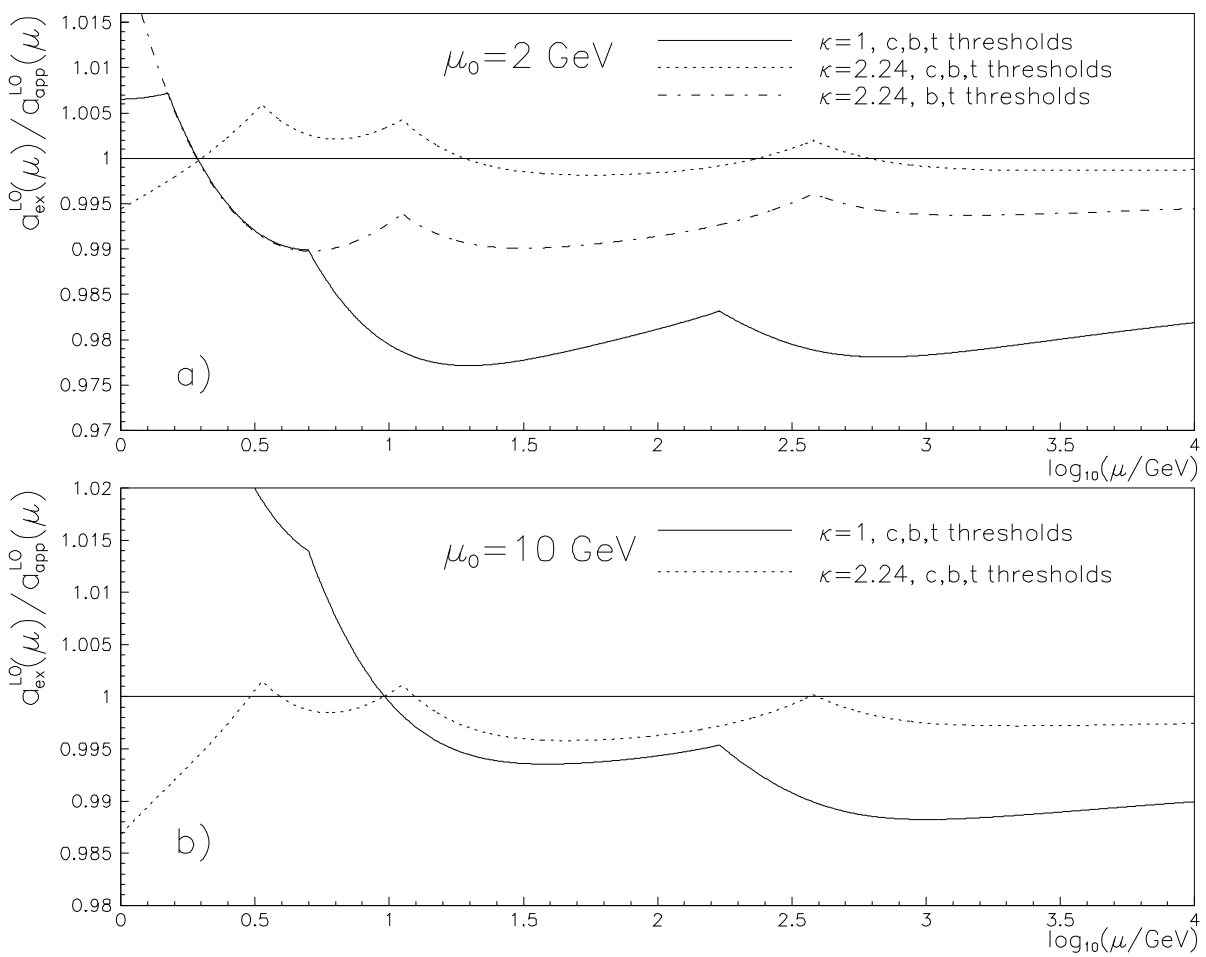

Figure 2: The same as in Fig. 1b, but for $\mu_{0}$ equal to 2 and $10 \mathrm{GeV}$.

- If for $\mu_{0}=2 \mathrm{GeV}$ and $\kappa=\sqrt{5}$ only the $b$ and $t$ quark thresholds (dash-dotted curve in Fig. 2a) are considered [3, the result is markedly further away from the the exact extrapolation than if also the $c$ quark threshold is taken into account. This illustrates the influence of the proper treatment of charmed quark threshold even at the energy scales around $M_{Z}$.

The influence of the choice of $\kappa$ on the results of the matching procedure has recently been investigated, within the approach introduced in [13], in [1]. Although this matching procedure is more sophisticated than that based on the relations (9)-(11) and is applicable at any order, it relates couplants corresponding to different numbers of massless quarks only and thus does not concern the difference between the results using the exact expression for the function $h(x)$ and those based on its step approximations, as investigated in this note. The conclusion reached there, i.e. that $\alpha_{s}\left(M_{Z}\right)$ obtained by extrapolation from low energy scales changes little (by less than $0.6 \%$ ) when the matching points are varied in some reasonable interval, is therefore not in contradiction with the result of this note.

In summary, the approximate treatment of quark mass thresholds, based on the step-like approximation of the QCD $\beta$-function and using the matching procedure of [11] with $\kappa=1$, leads to an overestimate of $\alpha_{s}^{\mathrm{LO}}\left(M_{Z}\right)$ extrapolated from low energy quantities, in particular the $R_{\tau}$ ratio, by about $2 \%$. Though not a large effect, it is of the same magnitude as the typical NNLO corrections to $\alpha_{s}^{\mathrm{NLO}}\left(\overline{\mathrm{MS}}, M_{Z}\right)$. This overestimate of the extrapolated $\alpha_{s}\left(M_{Z}\right)$ may explain part of the discrepancy between the extrapolations based on $R_{\tau}$ and deep inelastic scattering. On the other hand, the choice of $\kappa=\sqrt{5}$, suggested by theoretical considerations, yields extrapolations which are in much better agreement with those based on the exact form of the threshold function $h(x)$. The analysis of quark mass effects, presented above, holds strictly speaking, at the LO only. Nevertheless as both the mass effects the higher order perturbative corrections are small effects, it seems reasonable to expect that the main conclusions of the present analysis have more general

\footnotetext{
${ }^{3}$ In these circumstances and for $\kappa=\sqrt{5}$ the matching point corresponding to charmed quark, given by $\sqrt{5} m_{c} \doteq 3.26$ is below $\mu_{0}$.
} 
validity.

I am gratefull to P. Kolář and J. Rameš for carefull reading of the manuscript and useful comments and suggestions.

\section{References}

[1] W. Bernreuther: preprint PITHA 94/31

[2] D.V. Schirkov, S.V. Michailov: Z. f. Physik C63 (1994), 463

[3] S.G. Gorishny, A.L. Kataev, S.A. Larin: Phys. Lett. B259 (1991), 144

[4] S.G. Gorishny, A.L. Kataev, S.A. Larin: Phys. Lett. B259 (1991), 144

[5] S.A. Larin, J.A.M. Vermaseren: Phys. Lett. B259 (1991), 345

[6] S.A. Larin, T. van Ritbergen, J.A.M. Vermaseren: Phys. Lett. B320 (1994), 159

[7] S. Bethke: Talk presented at the QCD '94, Montpellier, France, July 1994, PITHA 94/30

[8] E. Braaten, S. Narison, A. Pich: Nucl. Phys. B373 (1992), 581

[9] A. Pich: FTUV/94-71, hep-ph/9412273

[10] M. Schifman: University of Minnesota preprint UMN-TH-1323-94, hep-ph/9501222

[11] W. Marciano: Phys. Rev. D29 (1984), 580

[12] A. De Rujula, H. Georgi: Phys. Rev. D13 (1976), 1296

[13] W. Bernreuther, W. Wetzel: Nucl. Phys. B197 (1982), 228 